\title{
Firmware Design for Portable PCR Devices Controlled by Smart Phones through Wireless Communication
}

\author{
Wan Yeon Lee ${ }^{1}$, Min Ja Kim², YoungWoong $\mathrm{Ko}^{3}$ and Jong Dae Kim${ }^{4,1}$ \\ ${ }^{1}$ Dept. of Computer Science, Dongduk Women's University, South Korea \\ ${ }^{2}$ Dept. of Computer Science and Engineering, Korea University, South Korea \\ ${ }^{3}$ Dept. of Computer Engineering, Hallym University, South Korea \\ ${ }^{4}$ Dept. of Convergence Software, Hallym University, South Korea ${ }^{4}$ \\ ${ }^{1}$ wanlee@dongduk.ac.kr, ${ }^{2}$ mjfeel@korea.ac.kr, ${ }^{3} y u k o @ h a l l y m . a c . k r$, \\ ${ }^{4}$ kimjd@hallym.ac.kr
}

\begin{abstract}
In this paper, we design and implement a firmware for portable PCR devices that is controlled by a smart phone. The firmware has the host-local structure in which the firmware receives operation commands from the smart phone and sends operation results to the smart phone through Bluetooth communication. The firmware is designed to accommodate unstable wireless communication of Bluetooth. We implement a low-cost small PCR device with the proposed firmware on microchip PIC18F4550, and verify that the implemented PCR device significantly reduces volume size of existing commercial $P C R$ devices with a similar performance.
\end{abstract}

Keywords: Firmware, PCR device, smart phone, Bluetooth, host-local structure

\section{Introduction}

Analyzing DNA characteristics of target materials requires four steps: DNA extraction, DNA amplification, electrophoresis, and gel documentation [1]. DNA extraction is a process of purification of DNA particles from target materials using a combination of physical and chemical methods. DNA amplification is a process of producing multiple copies of a sequence of DNA. PCR(Polymerase Chain Reaction) is the most common DNA amplification method [2]. Electrophoresis is a process of separating DNA fragments based on their size by moving dispersed DNA particles through agarose gel under influence of a spatially uniform electric field [3]. Gel documentation is a process of capturing a photograph of DNA particles dispersed on agarose gels under ultra violet light and analyzing the relative moving distance of DNA particles [4]. In this paper, we focus on designing an efficient device performing the PCR procedure for DNA amplification.

The proposed PCR structure minimizes the volume size and the implementation cost in order to support portability. The proposed structure supports only the PCR function but not the user interface function to minimize the volume size and the implementation cost. In the proposed structure called the host-local structure [5, 6], the user interface function is eliminated by exploiting the user interface function of smart phones via Bluetooth wireless communication. In this paper, we design and implement a firmware for microcontroller of PCR devices so that the PCR device would satisfy the following three requirements: receiving/sending the input/output from/to smart phones through wireless communication, the minimal volume size and low implementation cost, and reliable PCR

\footnotetext{
* Corresponding Author: Jong Dae Kim, email: kimjd@halym.ac.kr

This paper is a revised and expanded version of a paper entitled "Design of compact PCR device based on host-local structure" presented at International Conference on Next Generation Computer and Information Technology, Hochimin, October 2014.
} 
function. To minimize the code size, the proposed firmware schedules tasks using only hardware timer interrupts and excludes the support of light real-time OS such as PICos18, freeRTOS and microC/OS-ii.

To evaluate working reliability of the designed firmware, we implement the designed firmware on PIC18F4550 micro-controller chip and construct a prototype PCR device controlled by the designed firmware. Compared with a commercial PCR device, the prototype PCR device reduces the volume size by $6 / 7$ while maintaining the DNA amplification performance similarly.

Our previous studies [7, 8] addressed the design issues of low-cost small PCR devices. Whereas our previous studies handled the PCR structure controlled by PC through stable wired usb communication, this paper handles the PCR structure controlled by smart phones through unstable wireless Bluetooth communication. This paper does not consider a disposable microchip-based PCR device $[9,10]$.

The rest of this paper is organized as follows; Section 2 explains the background knowledge to understand the proposed scheme. Section 3 describes the proposed design in detail. Section 4 shows the evaluation results and Section 5 gives the concluding remarks.

\section{Preliminaries}

\subsection{PCR Protocol}

The protocol for Polymerase chain reaction (PCR) follows three steps: 1) denaturation to separate the two strands of DNA using heat, 2) annealing, where the primer combines to the end of the sequence that is targeted to be amplified while cooling down, 3) polymerization, of extension, synthesizing DNA through re-heating the sample. PCR thermal cycler controls the temperature of a metal block with holes for the samplecontaining tubes usually using Peltier thermoelectric devices. Table 1 is an example of a PCR protocol. The first low in the table, labeled as ' 1 ', is an extra denaturation step maintaining the chamber at $95^{\circ} \mathrm{C}$ for 30 seconds to easily separate the DNA strands before the normal cycling. The next three rows form one cycle of amplification, each representing denaturation, annealing and polymerization. 'GOTO' label is for the flow control, indicating the cycle to return to label 2 for 34 times. The example therefore consists of 35 cycles in total. The last row is an extra polymerization step finishing the PCR. This step enables the strands that has not bond yet to bond with their complementary strand.

Table 1. PCR Protocol Example

\begin{tabular}{|c|c|c|}
\hline label & Temperature $\left({ }^{\circ} \mathrm{C}\right)$ & Duration $(\mathrm{sec})$ \\
\hline 1 & 95 & 30 \\
\hline 2 & 95 & 30 \\
\hline 3 & 55 & 30 \\
\hline 4 & 72 & 30 \\
\hline GOTO & 2 & 34 \\
\hline 5 & 72 & 180 \\
\hline
\end{tabular}

\subsection{Hardware Components for PCR Operation}

The proposed PCR structure employs the host-local model $[5,6]$. In the host-local model, the system management functions such as UI functions and file handling functions are moved to an existing computing system, called host system. The newly manufactured 
system, called local system, possesses only basic functions except the system management functions. The host-local model has two main benefits. One is to conserve human and time resources by eliminating the implementation of the system management functions into the local system. The other is to reduce the volume size and the implementation cost of the local system. In this paper, a smart phone plays a role of the host system and we design a new device that plays a role of the local system. The newly manufactured system performs the PCR protocol and communicates with the smart phone via Bluetooth.

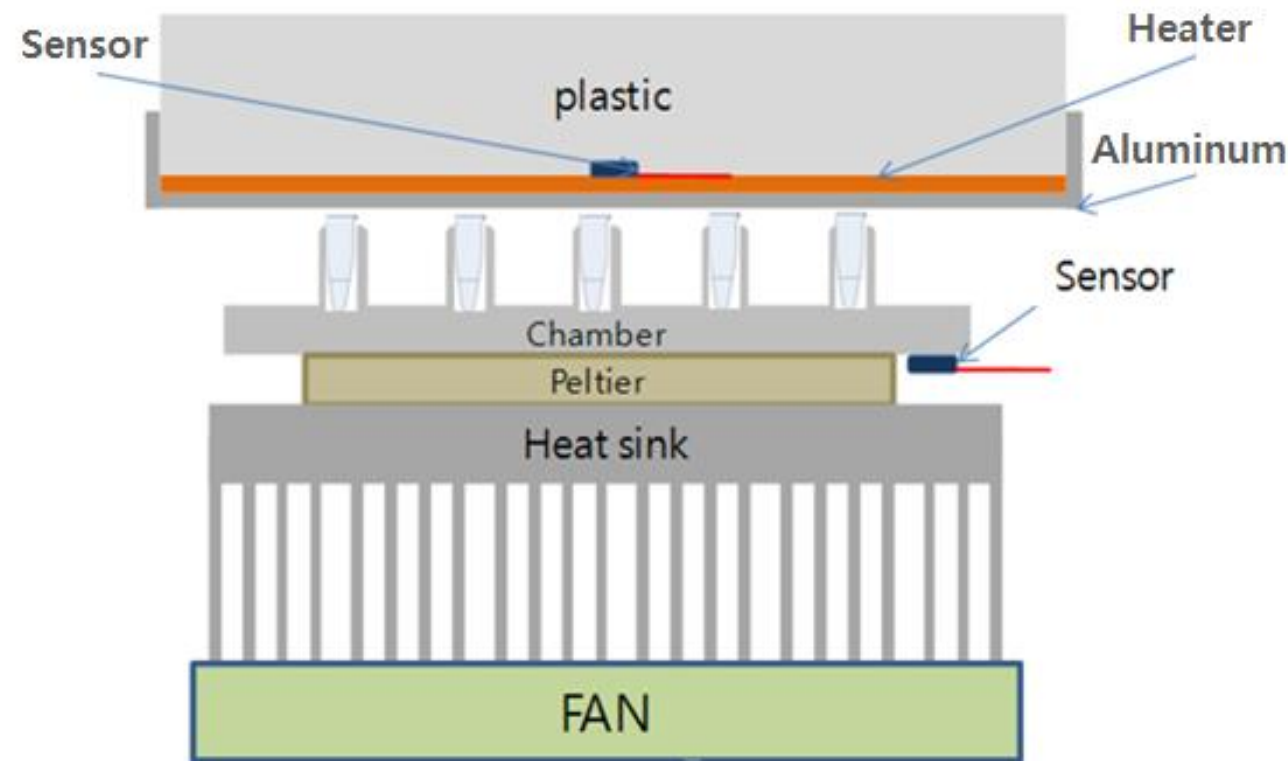

Figure 1. Configuration of Hardware Components

Figure 1 shows the configuration of hardware components that are controlled by a micro-controller and perform temperature changes for PCR protocols. The plastic in the top of the figure is the cover of this device. The sensor below the plastic measures the temperature data of chamber and transfers it to the micro-controller. The coil heater increases the temperature of chamber and the aluminum spreads the heat evenly. The chamber contains DNA fragments being amplified and reagents triggering PCR operation. The Peltier below the chamber absorbs or produces heats according to the flow direction of electric current. The heater sink and the fan in the bottom of the figure help the absorbed heat to emit outside. The detailed configuration and operation mechanism of each component are addressed in our previous study [7].

\section{Proposed Firmware Design}

The proposed firmware employs the host-local model as shown in Figure 2. Utilizing GUI (graphic user interface) on a smart phone, a user inputs a PCR protocol that holds temperature changes and their number of repetitions. The protocol is transferred to the firmware running on a micro-controller through Bluetooth wireless communication. After completely receiving the PCR protocol, the firmware starts to perform the temperature transitions in the PCR protocol. The firmware generates control signals of heating or cooling the Peltier component according to the temperature transitions in the PCR protocol. Meanwhile, the firmware collects the temperature data measured by sensors periodically. Based on the collected temperature data, the firmware determines the heating strength and the cooling strength to reach the target temperature of each transition of the PCR protocol. To display the processing status of the PCR protocol to users, the information of the current step of temperature transition, the progressed time, the current 
measured temperature, etc., is transferred to the smartphone app. through Bluetooth wireless communication.

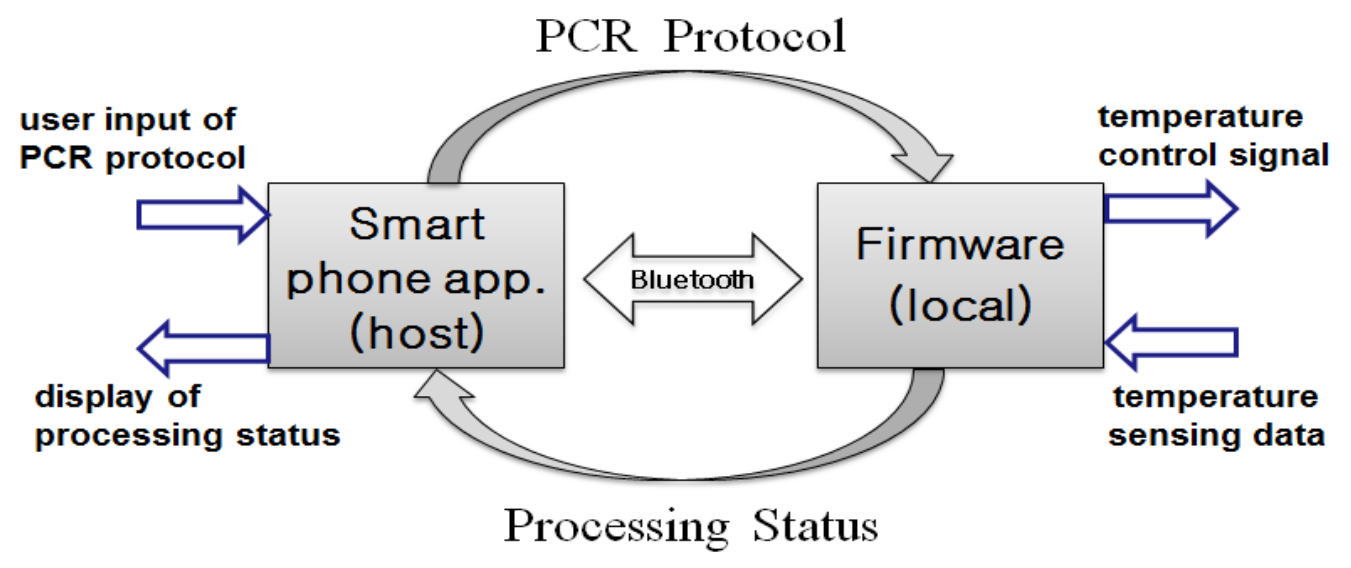

Figure 2. Host-local Structure

Figure 3 shows the state transition diagram of firmware on the local system. In 'start' state, the firmware waits to receive the start command for PCR operation. In 'protocol transferring' state, the firmware receives a PCR protocol composed of multiple messages from the host system. If the PCR protocol is completely transferred, the next state becomes 'execution ready'. If it fails to transfer the PCR protocol due to sudden disconnection of unstable wireless communication, the state goes back to the 'start' state. In 'execution ready' state, the firmware parses the transferred PCR protocol. In 'protocol execution' state, the firmware generates control signals of temperature heating or cooling according to the parsed target temperature and its duration of each step. Meanwhile, the firmware collects temperature data measured by sensors, and sends the collected temperature data to the host system. When the whole PCR protocol is finished, the next state becomes 'protocol completion'. In 'protocol completion' state, the firmware sends a notice of completion to the host system and waits a confirmation response from the host system. When the confirmation message arrives, the next state becomes 'start'.

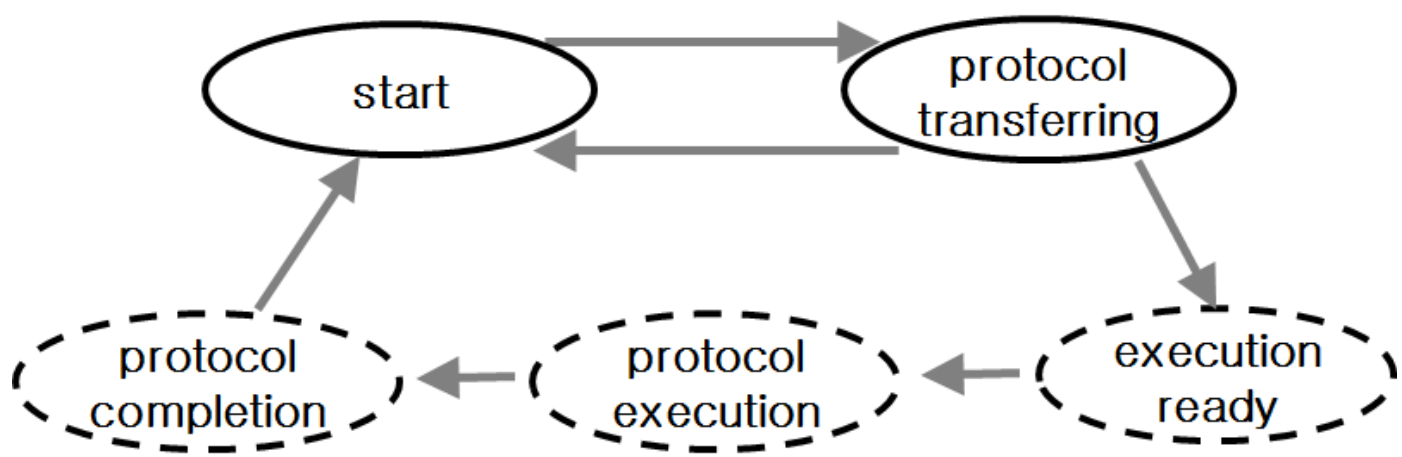

Figure 3. State Transition Diagram of Firmware

In the two states of 'start' and 'protocol transferring' depicted with solid lines, the firmware needs to hold the connection with the host system. If the connection is broken due to unstable wireless connection, the firmware does nothing excepting waiting to receive a message from the host system. On the contrary, in the three states of 'execution ready', 'protocol execution' and 'protocol completion' depicted with broken lines, the firmware works regardless of connection with the host system. In these states, the firmware does not need any command arrived from the host system and thus neglects any 
command even if some unexpected command arrives. When the connection is broken, the firmware just skips to send messages to the host system. The skipped messages are not essential for the host system because they include information for displaying the processing status of the firmware. In these three states, the host system displays the processing status if the connection is established and, otherwise, stops to display the processing status.

The firmware receives a message and sends its response message periodically. We determine the period empirically. In our experiments that will be explained in Chapter 4 , the maximum turn around delay is smaller than $200 \mathrm{~ms}$. This implies that the maximum delay of one-way communication is smaller than $100 \mathrm{~ms}$. Hence the firmware checks messages arrived from the host system per $100 \mathrm{msec}$. Also, if a message for the host system is ready, the firmware sends the message per $100 \mathrm{msec}$. In contrast, the host system checks arrived messages and sends a message to the local system with a different period. If the period for the host system is equal to that for the local system, messages are possibly lost even when the wireless connection is established, due to synchronization problem. The period for the host system is determined to be $200 \mathrm{msec}$. The display image updated every $200 \mathrm{msec}$. causes negligible inconvenience for users.

The host system and the local system have the same size of messages with 32 bytes. The former 8 bytes are used for commands information in the host system and processing status information in the local system. The rest 23 bytes are used to hold debugging information.

\section{Evaluation}

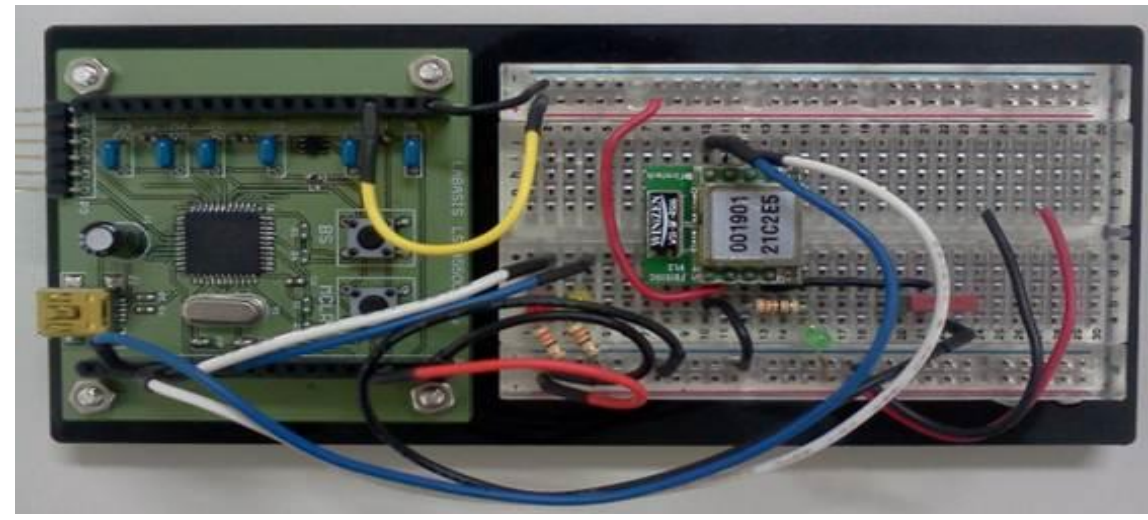

Figure 4. Test-bed micro-controller

To examine the maximum turn-around delay of Bluetooth wireless communication, we first construct a test-bed board shown in Figure 4. For Bluetooth communication, the FB155BC chip of Firmtech corporation is equipped. The maximum available distance in hardware specification of the chip is $10 \mathrm{~m}$. The firmware designed in Section 3 is developed and loaded in the microchip PIC18F4550 micro-controller. The firmware is implemented using MPLAB IDE, MPLAB c18 lite compiler, microchip application libraries, HIDBootloader program on 32bit Window 7 OS. The required size of flash memory for storing the binary execution code of the firmware is about 1 Kbytes, and the required size of RAM memory for storing the data of the firmware is about 7,000 bytes.

The test-bed board is actually not equipped with the hardware components, because it is used only for measurement of turn-around delay of Bluetooth wireless communications. The temperature control signals are not transmitted to the Peltier hardware component and the temperature data is virtually generated instead of measuring by sensors.

Table 2 shows the experimental results of measuring the turn-around delay of the testbed board shown in Figure 4. We perform 10,000 trials for each setting. Although the 
maximum distance in the hardware specification of FB155BC Bluetooth chip is $10 \mathrm{~m}$, the wireless connection is established between the test-bed board and the android smart phone apart by more than $10 \mathrm{~m}$. While no dropped message is dropped within $10 \mathrm{~m}$, more messages are dropped in a longer distance when the distance is larger than $10 \mathrm{~m}$. In the distance longer than $25 \mathrm{~m}$, most messages are dropped. The maximum turn-around delay is $145 \mathrm{msec}$ in $25 \mathrm{~m}$ distance. The average delay is approximately $35 \mathrm{msec}$.

Table 2. Turn-around Delay of Bluetooth Wireless Communication

\begin{tabular}{|c||c|c|c|c|c|}
\hline Distance & $\mathbf{5 m}$ & $\mathbf{1 0 m}$ & $\mathbf{1 5 m}$ & $\mathbf{2 0 m}$ & $\mathbf{2 5 m}$ \\
\hline \hline $\begin{array}{c}\text { Maximum } \\
\text { delay }\end{array}$ & $97 \mathrm{msec}$ & $82 \mathrm{msec}$ & $142 \mathrm{msec}$ & $141 \mathrm{msec}$ & $145 \mathrm{msec}$ \\
\hline $\begin{array}{c}\text { Average } \\
\text { delay }\end{array}$ & $33 \mathrm{msec}$ & $34 \mathrm{msec}$ & $36 \mathrm{msec}$ & $36 \mathrm{msec}$ & $37 \mathrm{msec}$ \\
\hline $\begin{array}{c}\text { Ratio of dropped } \\
\text { messages }\end{array}$ & $0 \%$ & $0 \%$ & $0.8 \%$ & $7.2 \%$ & $16.3 \%$ \\
\hline
\end{tabular}

Figure 5 shows the graphic user interface (GUI) of the implemented application running on a reference Android phone, Google Nexus 4 of LG Corporation. This application is implemented using JDK SE 1.7, Eclipse IDE for Java Developers, ADT plug-in, Android SDK 2.2 API on 32bit Window 7 OS. The left figure is the display image of PCT protocol input by users. The right figure is the display image of processing status for PCR protocol, which is transferred from the local system.
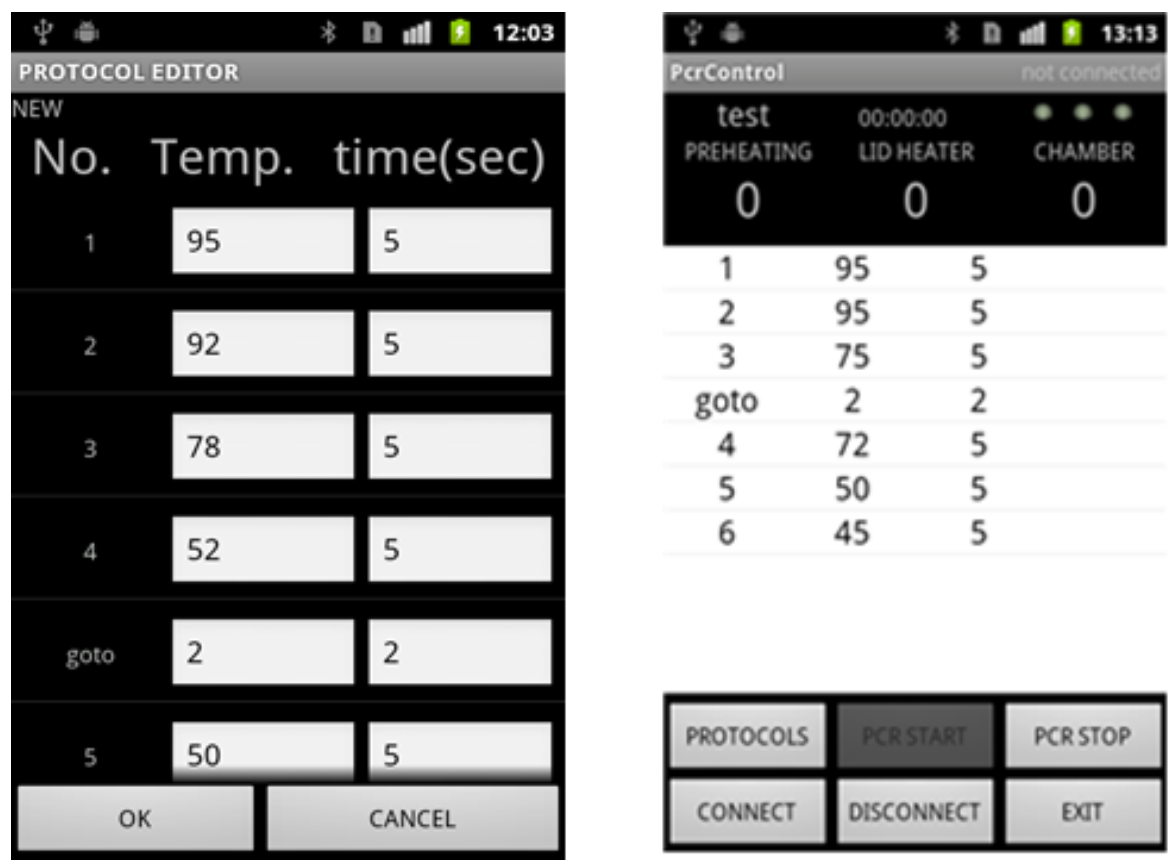

Figure 5. Graphic User Interface for Android Phones

For comparison of practical benefits, we construct a prototype PCR device in which the micro-controller shown in Figure 4 is connected to the hardware components explained in Section 3.2. The left figure in Figure 6 is the constructed PCR device. The antenna in the figure is for Bluetooth wireless communication. The right figure in Figure 6 is the existing commercial PCR product, MyGene PCR of the LongGene Corporation, which is known as the smallest one with the lowest price among commercial PCR products. The volume 
of the constructed PCR device is width $11.5 \mathrm{~cm} \times$ length $13.6 \mathrm{~cm} \times$ height $19 \mathrm{~cm}=2,971.6$. The volume of the existing PCR product is width $24 \mathrm{~cm} \times$ length $31.5 \mathrm{~cm} \times$ height $27.5 \mathrm{~cm}$ $=20,790$. The volume ratio of the constructed PCR device to the existing smallest PCR product is about $1 / 7$.

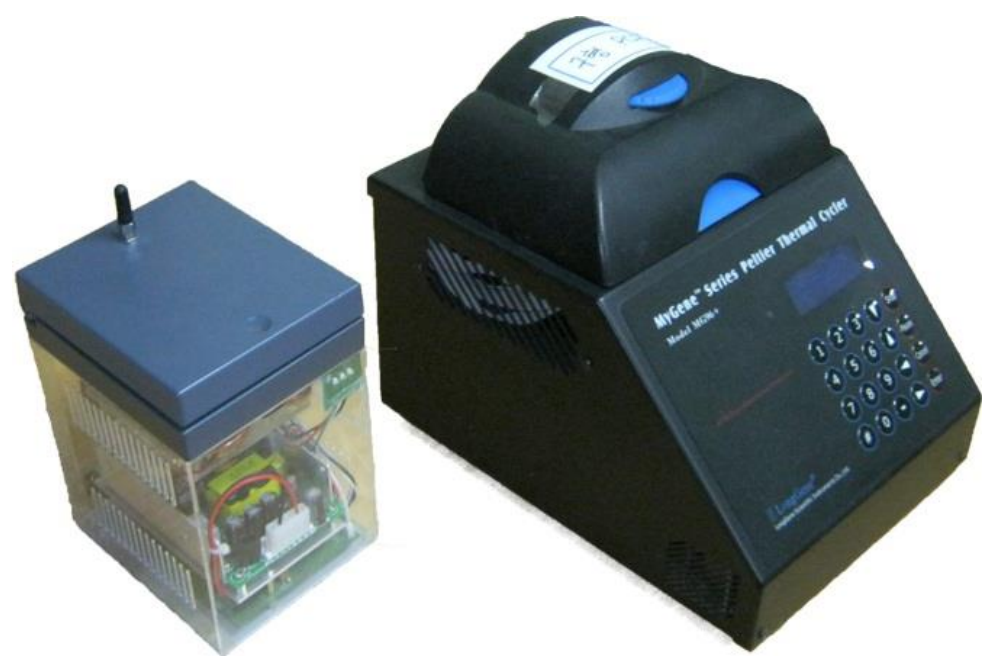

Figure 6. Volume Comparison

Figure 7 shows the results of DNA amplification performed by the constructed PCR device and the existing commercial PCR product shown in Figure 6. The DNA amplification results are analyzed using the same electrophoresis device and gel documentation system. In Figure 7, the left part shows the analyzed image of the existing commercial device, and the center part shows that for the constructed device. The two analyzed images are almost equal, which implies that their DNA amplification performances are almost equal. The right part in Figure 7 shows that the constructed PCR device does not amplify DNA fragments when the sample does not contain the target DNA fragments.

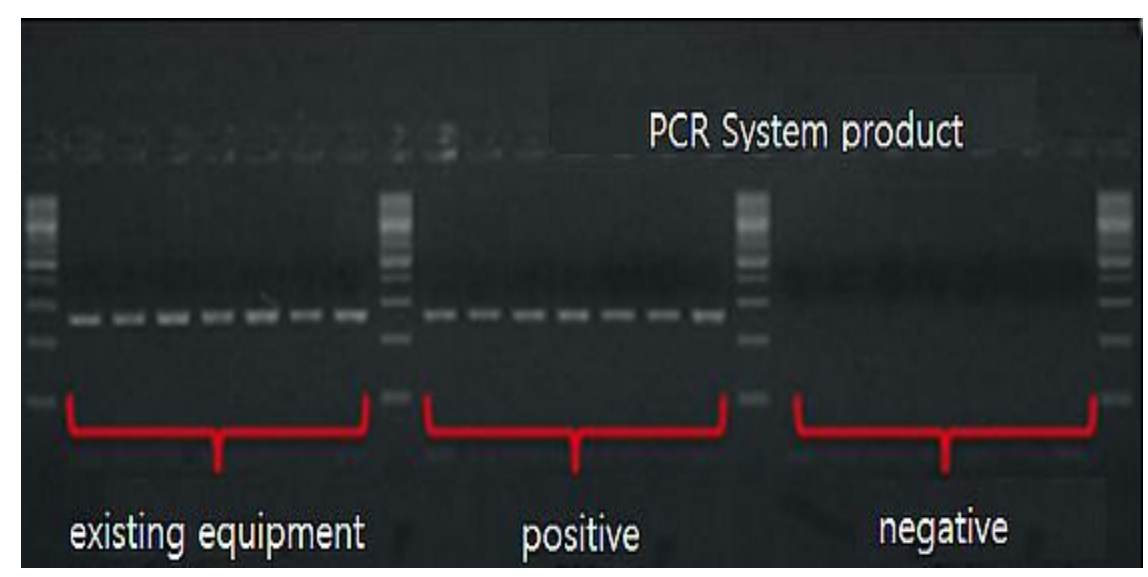

Figure 7. Results of PCR Amplification

\section{Conclusions}

We design a firmware for portable PCR device that is controlled by a smart phone through unstable Bluetooth wireless communication. The firmware is designed to stably perform DNA amplification under unstable arrivals of the operation command from the smart phone. Also the firmware is designed to be suitable for portable PCR device. The 
firmware can be loaded on a micro-controller with a size of 10 Kbytes memory. To verify the feasibility of the designed firmware, the firmware is actually implemented and loaded on a prototype PCR device equipped with a PIC18F4450 micro-controller chip and hardware components playing a role of heating or cooling. Evaluation results shows that the constructed prototype device enhances portability by significantly reducing the volume size with a similar performance of DNA amplification, compared with existing commercial PCR products.

\section{Acknowledgements}

The preliminary version of this paper was published in International Conference on Next Generation Computer and Information Technology, October 2014. This research was supported by Basic Science Research Program through the National Research Foundation of Korea(NRF) funded by the Ministry of Education, Science and Technology(2011-0009358).

\section{References}

[1] T. A. Brown, Gene Cloning and DNA Analysis: An Introduction, 6th Ed., Wiley-Blackwell, West Sussex (2010).

[2] R. Saiki, D. Gelfand, S. Stoffel, S. Scharf, R. Higuchi, G. horn, K. Mullis, H. Erlich, "Primer-directed enzymatic amplification of DNA with a thermostable DNA poylerase," Science (1988), vol. 239, no. 4839, pp. 487-491.

[3] B. Hames and D. Rickwood, Gel Electrophoresis of Proteins: a Practical Approach, 3rd Ed., Oxford University Press., New York (1998).

[4] T. Goldmann, A. Zyzik, S. Loeschke, W. Lindsay, and E. Vollmer, "Cost-effective Gel Documentation Using a Web-cam," Journal of Biochemical and Biophysical Methods (2001), vol. 50, no. 1, pp. 91-95.

[5] C. Plaisant, A. Rose, B. Shneiderman and A. J. Vanniamparampil, "Low Effort, High Payoff User Interface Reengineering” IEEE Software (1997), vol. 14, no. 4, pp. 66-71.

[6] J. D. Kim, Y. U. Lee and S. Kim, "Efficient Hardware-Software Partitioning for a Digital Dental X-Ray System" IEICE Trans. Fundamentals (2003), vol. E86-A, no. 4, pp. 859-865.

[7] C. Y. Park, J. D. Kim, Y. S. Kim, H. J. Song, J. M. Kim and J. Kim, "Cost Reduction of PCR Thermal Cycler," International Journal of Multimedia and Ubiquitous Engineering (2012), vol. 7, no. 2, pp. 389394.

[8] W. Y. Lee and J. D. Kim, "Design and Implementation of Firmware for Low-cost Small PCR Devices," Journal of the Korean Society of Computer and Information (2013), vol. 18, no. 6, pp. 1-8 (in Korean).

[9] K. Shen, X. Chen, M. Guo and J. Cheng, "A microchip-based PCR device using flexible printed circuit technology," Sensors and Actuators B (2005), vol. 105, pp. 251-258.

[10] J. D. Kim, C. Y. Park, S.Y. Kim, O.D. Gwak, D.J. Lee, Y. S. Kim, and H. J. Song, "Chamber Temperature Measurement of Micro PCR Chip Using Thermocouple," International Journal of Multimedia and Ubiquitous Engineering (2012), vol. 7, no. 2, pp. 395-402.

\section{Authors}

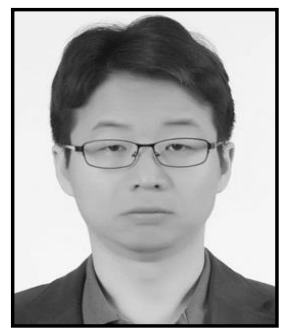

Wan Yeon Lee, he received the BS, MS, and $\mathrm{PhD}$ degrees in computer science and engineering from POSTECH in 1994, 1996, and 2000, respectively. From 2000 to 2003, he was a research engineer in LG electronics. He is an associate professor in the Department of Computer Science, Dongduk Women's University. His recent interests focus on embedded system, bioinformatics, computer security, and mobile computing. 


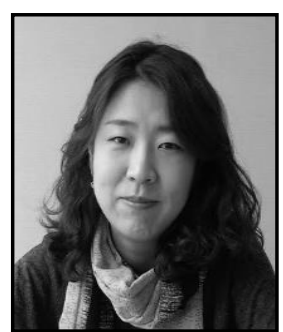

Min Ja Kim, she received the B.S. degree in computer engineering from Dongduk Women's University in 2000 and M.S. degree in Computer Science from Korea University in 2002. She is currently pursuing her Ph.D degree in College of Information and Communications, Korea University, Seoul, Korea. Her research interests include Operating System, File system and multimedia streaming.

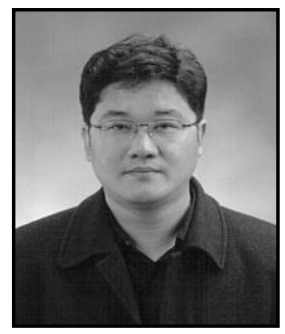

Young Woong Ko, he received both a M.S. and Ph.D. in computer science from Korea University, Seoul, Korea, in 1999 and 2003, respectively. He is now a professor in Department of Computer engineering, Hallym University, Korea. His research interests include operating system, embedded system and multimedia system.

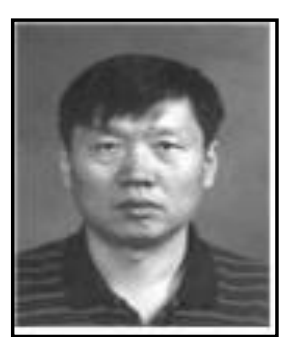

Jong Dae Kim, he received the M.S. and the Ph.D. degrees in Electrical Engineering from Korea Advanced Institute of Science and Technology, Seoul, Korea, in 1984 and 1990, respectively. He worked for Samsung Electronics from 1988 to 2000 as an electrical engineer. He is a Professor in Department of Convergence Software, Hallym University. His recent interests focus on biomedical system and bioinformatics. 
International Journal of Control and Automation Vol. 7, No. 11 (2014) 